\title{
THERMO-DIFFUSION AND HALL EFFECT ON RADIATING AND REACTING MHD CONVECTIVE HEAT ABSORBING FLUID PAST AN EXPONENTIALLY ACCELERATED VERTICAL POROUS PLATE WITH RAMPED TEMPERATURE
}

\author{
B. Prabhakar Reddy \\ Department of Mathematics \& Statistics, CNMS, The University of Dodoma, Dodoma, \\ Tanzania; \\ Department of Mathematics, Geethanjali College of Engineering \& Technology, Telangana, \\ India \\ e-mail: prabhakar.bijjula@gmail.com
}

\begin{abstract}
Numerical investigation is undertaken to study the effects of thermo-diffusion and Hall current on unsteady magneto-hydrodynamic convective flow of a viscous, incompressible, electrically conducting, radiating and heat absorbing fluid past an exponentially accelerated infinite vertical porous plate with ramped temperature in the presence of chemical reaction. A uniform magnetic field is applied transversely in the direction of the flow. The governing system of partial differential equations along with initial and boundary conditions is transformed to dimensionless form and then solved by employing finite element method. The impact of various flow parameters on the primary and secondary fluid velocities, fluid temperature and fluid concentration as well as shear stress, rate of heat and mass transfer at the plate are displayed through the graphs and tables. It can be observed that the temperature profiles are slower in case of ramped temperature plate than that of isothermal plate.
\end{abstract}

Keywords: Hall current, MHD, radiation, chemical reaction, heat absorption, vertical porous plate.

\section{Introduction}

The problem of magneto-hydrodynamic (MHD) with heat and mass transfer in the presence of radiation and thermo-diffusion (Soret) effects has attracted the attention of many researchers due to its diverse applications. The Soret effect is a phenomenon where light, medium and heavy molecules separate under the temperature gradient. Usually, this effect is important where more than one chemical species is present under a very large temperature gradient. The problems of this kind are very useful for isotope separation and in mixture between gasses with very light molecular weight. In several engineering processes, it occurs at high temperature where the knowledge of radiative heat transfer becomes indispensable such as the design of pertinent equipment, nuclear power plants, gas turbines and various propulsion devices for aircraft, missiles, satellites, and space vehicles. Radiation effects on free convection flow past a semiinfinite vertical plate were investigated by Soundalgekhar and Takhar (1993). Takhar et al. (1996) 
analyzed radiation effects on MHD free convection flow of a radiating gas past a semi-infinite vertical plate. Makinde (2005) studied free convection flow with thermal radiation and mass transfer past a moving vertical plate. Radiation effects on MHD flow past an impulsively started infinite vertical plate through a porous medium with variable temperature and mass diffusion were elucidated by Shanker and Gnaneshwar (2007). Mahmoud (2009) examined the thermal radiation effects on unsteady MHD free convection flow past a vertical plate with temperature dependent viscosity. The effects of thermal radiation on unsteady mixed convection flow and heat transfer over a porous stretching surface in porous medium was analyzed by Mukhopadhyay (2009). Rao and Reddy (2010) studied heat and mass transfer of an unsteady MHD natural convection flow of a rotating fluid past a vertical porous plate in the presence of radiative heat transfer. Shanker et al. (2010) investigated radiation and mass transfer effects on an unsteady MHD free convective fluid flow embedded in a porous medium with heat generation/absorption. Radiation effects on MHD flow through a porous medium with variable temperature and mass diffusion were examined by Rajesh and Varma (2010). Muralidhran and Muthucumaraswamy (2013) presented radiation effects on linearly accelerated plate with variable mass diffusion in the presence of magnetic field. Unsteady MHD free convection flow with Hall Effect of a radiating and heat absorbing fluid past a moving vertical plate with variable ramped temperature were studied by Seth et al. (2016). Recently, Reddy and Sunzu (2018) investigated Dufour and Soret effects on unsteady MHD free convection flow of viscous incompressible fluid past an infinite vertical porous plate in the presence of radiation.

The problem of chemical reaction effects on MHD heat and mass transfer flow arises in many chemical engineering processes. A chemical reaction occurs between a foreign mass and the fluid in which the plate is moving. These processes take place in numerous industrial applications such as manufacturing of ceramics and glassware, polymer production and food processing. Mahapatra et al. (2010) presented the effects of chemical reaction on free convection flow through a porous medium bounded by a vertical surface. Makinde and Olanrewaju (2011) studied unsteady mixed convection with Soret and Dufour effects past a porous plate moving through a binary mixture of chemically reacting fluid. The unsteady magneto-hydrodynamic convective heat and mass transfer past an infinite vertical plate in a porous medium with radiation, heat generation/absorption and chemical reaction were reported by Shateyi and Motsa (2011). Rajesh (2012) analyzed the effects of mass transfer on the flow past an impulsively started infinite vertical plate with Newtonian heating and chemical reaction. Venkateswarlu et al. (2013) investigated the effects of chemical reaction and heat generation on MHD boundary layer flow of a moving vertical plate with suction and viscous dissipation. Venkateswarlu and Padma (2015) studied unsteady MHD free convective heat and mass transfer in a boundary layer flow past a vertical permeable channel with thermal radiation and chemical reaction. Kumar et al. (2016) examined the thermal diffusion and chemical reaction effects on unsteady flow past a vertical porous plate with heat source in the slip flow regime. Prakash et al. (2016) analyzed diffusionthermo effects on MHD free convective radiative and chemically reactive boundary layer flow through a porous medium over a vertical plate. Thermal diffusion, Hall current and chemical reaction effects on unsteady MHD natural convection flow past a vertical plate analyzed by Venkateswarlu et al. (2017). Recently, Reddy and Peter (2019) presented the effects of chemical reaction on MHD flow past an impulsively started infinite vertical plate with variable temperature and mass diffusion in the presence of Hall current by finite difference method. Radiation and chemical reaction effects on unsteady MHD free convection parabolic flow past an isothermal infinite vertical plate with viscous dissipation was examined by Reddy (2019).

The recent research for applications of MHD is towards a strong magnetic field, due to which the impact of Hall current become very important. Actually, when the density of an electrically conducting fluid is low and/or applied magnetic field is strong, Hall current is produced in the flow-field, which plays an important role in determining flow features of the problems since it 
induces secondary flow in the flow-field. Applications of MHD boundary layer flow under the effect of Hall current are encountered in MHD power generators and pumps, Hall accelerators, refrigeration coils, electric transformers, in flight MHD, solar physics, the structure of the magnetic stars, oil extraction, thermal energy storage and flow through filtering devices and porous material regenerative heat exchangers. Sharma et.al (2007) investigated the effect of Hall current on MHD mixed convective flow of a viscous incompressible fluid past a vertical porous plate immersed in a porous medium with heat source/sink. Saha et. al (2011) presented the effect of Hall current on MHD natural convection flow from vertical permeable flat plate with uniform surface heat flux Reddy and Rao (2011) analyzed radiation and thermal diffusion effects on an unsteady MHD free convection mass transfer flow past an infinite vertical porous plate with Hall current and heat source by finite element method. Effects of Hall current on unsteady MHD flow between stretching sheet and an oscillatory porous upper parallel plate with constant suction was elucidated by Raju et al. (2011). Das et al. (2013) examined the Hall effect on MHD free convection boundary layer flow past a vertical flat plate. Das et al. (2016) analyzed the Hall effect on an unsteady magneto-convection and radiative heat transfer past a porous plate. Hall effect on unsteady MHD natural convective flow past an impulsively moving plate with ramped temperature and concentration were examined by Das et al. (2016). Rajput and Kanaujia (2016) studied MHD flow past a vertical plate with variable temperature and mass diffusion in the presence of Hall current. Seth et al. (2017) analyzed the effect of Hall current on MHD natural convection heat and mass transfer flow of rotating fluid past a vertical plate with ramped wall temperature.

The present work aims to examine the thermo-diffusion and Hall effects on the unsteady MHD convective boundary layer flow of radiating, reacting and heat absorbing fluid past an exponentially accelerated vertical porous plate with ramped temperature by finite element method. The influence of various control parameters on the primary and secondary fluid velocities, fluid temperature, fluid concentration, shear stress, rate of heat and mass transfer at the plate are presented through the graphs and tables and then discussed. To validate the present numerical procedure, a comparison was made between the present results and those of Seth et al. (2016) by considering the Nusselt number, it was noticed that an excellent agreement in this comparison. This provides the accuracy of present results.

\section{Mathematical Analysis}

Let us consider the unsteady magneto-hydrodynamic (MHD) convective flow of viscous, incompressible, electrically conducting, radiating and heat absorbing fluid past an exponentially accelerated infinite vertical porous plate with ramped temperature. A homogeneous first order chemical reaction is also considered. In the Coordinate system (Fig. 1), the $x^{\prime}$-axis is measured along the plate in the vertically upward direction, the $y^{\prime}$-axis measured normal to the plane of the plate which is directed into the fluid region and the $z^{\prime}-$ axis is measured normal to the $x^{\prime} y^{\prime}-$ plane. Since the plate is infinite in extent along $x^{\prime}$ and $z^{\prime}$-directions, so all the physical quantities except the pressure are the functions of $y^{\prime}$ and $t^{\prime}$ only. A uniform transverse magnetic field of strength $B_{0}$ is applied perpendicular to the plate in a direction parallel to $y^{\prime}$-axis. Initially, $t^{\prime} \leq 0$, both plate and fluid are at rest with constant temperature $T_{\infty}^{\prime}$ and concentration $C_{\infty}^{\prime}$. At time $t^{\prime}>0$, the plate accelerates exponentially along the $x^{\prime}$-axis with a velocity $U\left(t^{\prime}\right)=\exp \left(a^{\prime} t^{\prime}\right)$, where $a^{\prime}$ being an arbitrary constant. At the same time the temperature of the 
plate raised to $T_{\infty}^{\prime}+\left(T_{w}^{\prime}-T_{\infty}^{\prime}\right)\left(\frac{t^{\prime}}{t_{0}}\right)$ when $0 \leq t^{\prime} \leq t_{0}$ and maintained at $T_{w}^{\prime}$ when $t^{\prime}>t_{0}$, where $t_{0}$ being a critical time for rampedness, and the concentration maintained at $C_{\infty}^{\prime}+\left(C_{w}^{\prime}-C_{\infty}^{\prime}\right) A t^{\prime}$.

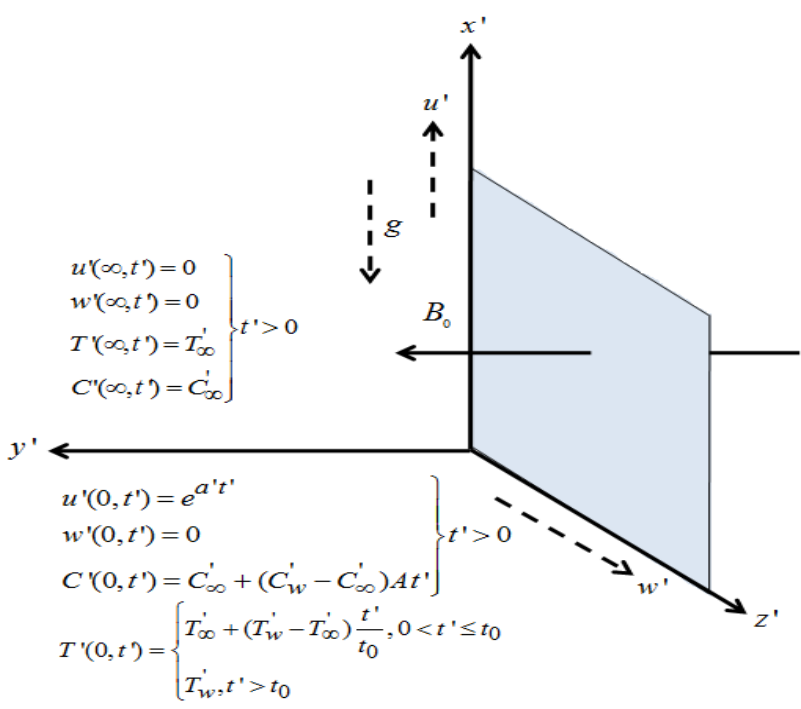

Fig. 1. Geometry of the physical problem.

The induced magnetic field produced by the fluid motion is negligible in comparison to the applied magnetic field. This assumption is acceptable because the magnetic Reynolds number is very small for liquid metals and partially ionized fluids, which are generally used in several industrial processes. The effect of polarization is neglected which corresponds to the case where no energy is added or extracted from the fluid by electrical means. In view of the above assumptions and under the usual Boussinesq's approximation, governing partial differential equations of the flow are:

$$
\begin{gathered}
\frac{\partial u^{\prime}}{\partial t^{\prime}}=v \frac{\partial^{2} u^{\prime}}{\partial y^{\prime 2}}-\frac{\sigma B_{0}^{2}}{\rho\left(1+m^{2}\right)}\left(u^{\prime}+m w^{\prime}\right)-\frac{v u^{\prime}}{K^{\prime}}+g \beta\left(T^{\prime}-T_{\infty}^{\prime}\right)+g \beta^{*}\left(C^{\prime}-C_{\infty}^{\prime}\right) \\
\frac{\partial w^{\prime}}{\partial t^{\prime}}=v \frac{\partial^{2} w^{\prime}}{\partial y^{\prime 2}}+\frac{\sigma B_{0}^{2}}{\rho\left(1+m^{2}\right)}\left(m u^{\prime}-w^{\prime}\right)-\frac{v w^{\prime}}{K^{\prime}} \\
\frac{\partial T^{\prime}}{\partial t^{\prime}}=\frac{k}{\rho c_{p}} \frac{\partial^{2} T^{\prime}}{\partial y^{\prime 2}}-\frac{1}{\rho c_{p}} \frac{\partial q_{r}}{\partial y^{\prime}}-\frac{Q_{0}}{\rho c_{p}}\left(T^{\prime}-T_{\infty}^{\prime}\right)+\frac{Q_{1}}{\rho c_{p}}\left(C^{\prime}-C_{\infty}^{\prime}\right) \\
\frac{\partial C^{\prime}}{\partial t^{\prime}}=D \frac{\partial^{2} C^{\prime}}{\partial y^{\prime 2}}+\frac{D K_{T}}{T_{m}} \frac{\partial^{2} T^{\prime}}{\partial y^{\prime 2}}-k_{r}^{\prime}\left(C^{\prime}-C_{\infty}^{\prime}\right)
\end{gathered}
$$

with the initial and boundary conditions: 


$$
\begin{aligned}
& u^{\prime}=0, w^{\prime}=0, T^{\prime}=T_{\infty}^{\prime}, C^{\prime}=C_{\infty}^{\prime} \text { for all } y^{\prime} \geq 0 \text { and } t^{\prime} \leq 0 \\
& u^{\prime}=e^{a^{\prime} t^{\prime}}, w^{\prime}=0, C^{\prime}=C_{\infty}^{\prime}+\left(C_{w}^{\prime}-C_{\infty}^{\prime}\right) A t^{\prime} \text { at } y^{\prime}=0 \text { for } t^{\prime}>0 \\
& T^{\prime}=T_{\infty}^{\prime}+\left(T_{w}^{\prime}-T_{\infty}^{\prime}\right) \frac{t^{\prime}}{t_{0}} \text { when } 0<t^{\prime} \leq t_{0} \text { at } y^{\prime}=0 \text { for } t^{\prime}>0 \\
& T^{\prime}=T_{w}^{\prime} \text { when } t^{\prime}>t_{0} \text { at } y^{\prime}=0 \text { for } t^{\prime}>0 \\
& u^{\prime} \rightarrow 0, w^{\prime} \rightarrow 0, T^{\prime} \rightarrow T_{\infty}^{\prime}, C^{\prime} \rightarrow C_{\infty}^{\prime} \text { as } y^{\prime} \rightarrow \infty \text { for } t^{\prime}>0
\end{aligned}
$$

where $\quad u^{\prime}, w^{\prime}, T^{\prime}, C^{\prime}, g, \beta, \beta^{*}, T_{\infty}^{\prime}, T_{w}^{\prime}, C_{\infty}^{\prime}, C_{w}^{\prime}, K^{\prime}, D, k, v, \rho, c_{p}, q_{r}, K_{T}, T_{m}, k_{r}^{\prime} \quad$ and $\quad t^{\prime} \quad$ are, respectively, fluid velocity in $x^{\prime}$-direction, fluid velocity in $z^{\prime}$-direction, fluid temperature, fluid concentration, acceleration due to gravity, volumetric coefficient of thermal expansion, volumetric coefficient of concentration expansion, free stream temperature, temperature of the fluid away from the plate, free stream concentration, concentration of the fluid away from the plate, permeability of the medium, mass diffusion, thermal conductivity, kinematic viscosity, fluid density, specific heat at constant pressure, radiation heat flux along $y^{\prime}$-direction, thermodiffusion rate, mean fluid temperature, chemical reaction rate and time.

The radiation heat flux $q_{r}$ for an optically low thick gray fluid under the Rosseland approximation is given by

$$
q_{r}=-\frac{4 \sigma^{\bullet}}{3 k^{*}} \frac{\partial T^{\prime 4}}{\partial y^{\prime}}
$$

where $\sigma^{\bullet}$ and $k^{*}$ are, respectively, the Stefan-Boltzmann constant and the mean absorption coefficient. Assuming a small temperature difference between $T^{\prime}$ and $T_{\infty}^{\prime}, T^{\prime 4}$ is expanding in a Taylor series about $T_{\infty}^{\prime}$, after neglecting second and higher order terms, one obtain

$$
T^{\prime 4}=4 T_{\infty}^{\prime 3} T^{\prime}-3 T_{\infty}^{\prime 4}
$$

On using Eqs. (6) and (7), Eq. (2), reduces to

$$
\frac{\partial T^{\prime}}{\partial t^{\prime}}=\frac{k}{\rho c_{p}}\left(1+\frac{16 \sigma^{\bullet} T_{\infty}^{\prime 3}}{3 k k^{*}}\right) \frac{\partial^{2} T^{\prime}}{\partial y^{\prime 2}}-\frac{Q_{0}}{\rho c_{p}}\left(T^{\prime}-T_{\infty}^{\prime}\right)+\frac{Q_{1}}{\rho c_{p}}\left(C^{\prime}-C_{\infty}^{\prime}\right)
$$

Let us introduce the following dimensionless parameters and quantities:

$$
\begin{aligned}
& u=\frac{u^{\prime}}{U_{0}}, w=\frac{w^{\prime}}{U_{0}}, \eta=\frac{y^{\prime} U_{0}}{v}, t=\frac{t^{\prime} U_{0}}{v}, S_{c}=\frac{v}{D}, P_{r}=\frac{\mu c_{p}}{k}, M^{2}=\frac{\sigma B_{0}{ }^{2} v}{\rho U_{0}^{2}}, K=\frac{K^{\prime} U_{0}^{2}}{v^{2}}, \\
& \gamma=\frac{k_{r}^{\prime} v}{U_{0}^{2}}, N_{r}=\frac{16 \sigma^{\circ} T_{\infty}^{\prime 3}}{3 k k^{*}}, Q_{H}=\frac{Q_{0} v}{\sigma c_{p} U_{0}^{2}}, t_{1}=\frac{t_{0} U_{0}^{2}}{v}, \theta=\frac{\left(T^{\prime}-T_{\infty}^{\prime}\right)}{\left(T_{w}^{\prime}-T_{\infty}^{\prime}\right)}, \phi=\frac{\left(C^{\prime}-C_{\infty}^{\prime}\right)}{\left(C_{w}^{\prime}-C_{\infty}^{\prime}\right)}, \\
& G_{r}=\frac{g \beta v\left(T_{w}^{\prime}-T_{\infty}^{\prime}\right)}{U_{0}^{3}}, G_{m}=\frac{g \beta^{*} v\left(C_{w}^{\prime}-C_{\infty}^{\prime}\right)}{U_{0}^{3}}, A=\frac{U_{0}^{2}}{v}, a=\frac{a^{\prime} v}{U_{0}^{3}}, S_{r}=\frac{D K_{T}\left(T^{\prime}-T_{\infty}^{\prime}\right)}{T_{m} v\left(C_{w}^{\prime}-C_{\infty}^{\prime}\right)}, \\
& R=\frac{Q_{1} v\left(C_{w}^{\prime}-C_{\infty}^{\prime}\right)}{\sigma c_{p} U_{0}^{2}\left(T_{w}^{\prime}-T_{\infty}^{\prime}\right)}
\end{aligned}
$$


into Eqs. (1), (2), (4) and (8), the following dimensionless governing system of partial differential equations of the flow are obtained:

$$
\begin{gathered}
\frac{\partial u}{\partial t}=\frac{\partial^{2} u}{\partial \eta^{2}}-\frac{M^{2}}{1+m^{2}}(u+m w)-\frac{u}{K}+G_{r} \theta+G_{m} \phi \\
\frac{\partial w}{\partial t}=\frac{\partial^{2} w}{\partial \eta^{2}}+\frac{M^{2}}{1+m^{2}}(m u-w)-\frac{w}{K} \\
\frac{\partial \theta}{\partial t}=\frac{\left(1+N_{r}\right)}{P_{r}} \frac{\partial^{2} \theta}{\partial \eta^{2}}-Q_{H} \theta+R \phi \\
\frac{\partial \phi}{\partial t}=\frac{1}{S_{c}} \frac{\partial^{2} \phi}{\partial \eta^{2}}+S_{r}\left(\frac{\partial^{2} \theta}{\partial \eta^{2}}\right)-\gamma \phi
\end{gathered}
$$

where $u, w, \theta, \phi, G_{r}, G_{m}, M, K, P_{r}, N_{r}, Q_{H}, R, S_{c}, S_{r}$ and $\gamma$ are respectively, dimensionless primary velocity, dimensionless secondary velocity, dimensionless temperature, dimensionless concentration, thermal Grashof number, mass Grashof number, magnetic parameter, permeability parameter, Prandtl number, radiation parameter, heat absorption parameter, radiation absorption parameter, Schmidt number, Soret number and chemical reaction parameter.

The initial and boundary conditions $(5 a)-(5 e)$ in dimensionless form become:

$$
\begin{array}{ll}
u=0, w=0, \theta=0, \phi=0 \text { for all } \eta \geq 0 \text { and } t \leq 0 & \text { a) } \\
u=e^{a t}, w=0, \phi=t \text { at } \eta=0 \text { for } t>0 & \text { b) } \\
\theta=\frac{t}{t_{1}} \text { when } 0<t \leq t_{1} \text { at } \eta=0 \text { for } t>0 & \text { c) } \\
\theta=1 \text { when } t>t_{1} \text { at } \eta=0 \text { for } t>0 & \text { d) } \\
u \rightarrow 0, w \rightarrow 0, \theta \rightarrow 0, \phi \rightarrow 0 \text { as } \eta \rightarrow \infty \text { for } t>0 & \text { e) }
\end{array}
$$

\section{Solution of the problem}

The finite element method has been applied to solve dimensionless governing system of coupled partial differential equations (10) to (13), subject to the initial and boundary conditions $(14 a)$ to $(14 e)$. Finite element method is an efficient numerical and computer based procedure to solve many engineering problems such as fluid mechanics, heat transfer, electrical systems, solid mechanics and chemical engineering, etc. Detailed discussion of the numerical procedure was given in Prabhakar Reddy (2019). Comprehensive review of the finite element method in the form of books was presented in Reddy (1985) and Bathe (1996). Thus, adopting this numerical procedure, a computer program was set up for the solutions of primary and secondary fluid velocities $u$ and $w$, fluid temperature $\theta$ and fluid concentration $\phi$. Numerical computations have been carried out to determine the impact of various involved physical parameters such as $G_{r}, G_{m}, M, m, K, P_{r}, N_{r}, Q_{H}, R, S_{c}, S_{r}$ and $\gamma$ on the primary and secondary fluid velocities $u$ and $w$, temperature $\theta$ and concentration $\phi$ as well as primary and secondary shear stresses $\tau_{x}$ and $\tau_{z}$, Nusselt number $N_{u}$ and Sherwood number $S_{h}$. The region of integration of the governing equations is divided into rectangular meshes formed by two sets of lines parallel to the coordinate 
axis. The region of integration is considered as a rectangle with sides $t_{\max }=2.5$ and $\eta_{\max }=4$ where $\eta_{\max }$ corresponds to $(\eta=\infty)$, which lies very well outside the momentum, thermal and the concentration boundary layers. The linear shape function was used to approximate the unknown fields $u, w, \theta$ and $\phi$. The mesh size $h=0.25$ in the space direction and $k=0.0625$ in the time direction was selected after comparing the results when the code was run with smaller values of $k=0.001,0.0009$ where it is noticed that the difference between these values is less than half a unity in the fourth decimal place. Also, it was found that the absolute difference between the values of $u, w, \theta$ and $\phi$ for two consecutive time steps is less than $10^{-5}$ at all nodal points. This designates that the method is stable. To judge the convergence of the method, computations are carried out by making small changes in the values of $h$ and $k$, no significant change was noticed in the values of $u, w, \theta$ and $\phi$. Hence, we conclude that the present method is stable and convergent.

Primary and secondary shear stresses at the plate $(\eta=0)$ are given by

$$
\tau_{x}=-\left(\frac{\partial u}{\partial \eta}\right)_{\eta=0} \text { and } \tau_{z}=-\left(\frac{\partial w}{\partial \eta}\right)_{\eta=0}
$$

Heat and mass transfer rate at the plate $(\eta=0)$ are given by

$$
N_{u}=-\left(\frac{\partial \theta}{\partial \eta}\right)_{\eta=0} \text { and } S_{h}=-\left(\frac{\partial \phi}{\partial \eta}\right)_{\eta=0}
$$

\section{Validation of the solution}

In order to verify the accuracy of the present solution, we have considered the analytical solution obtained by Seth et. al (2016) and computed the numerical results for Nusselt number $N_{u}$ in the absence of radiation absorption parameter $(R=0)$. These computed numerical results are presented in Tables 1 and 2. From these tables, it has been noticed that the present results are in good agreement with the results of Seth et.al (2016) obtained by Laplace transform technique. This justifies the correctness of the results presented in the paper.

\begin{tabular}{ccccccc}
\hline & \multicolumn{3}{c}{$\begin{array}{c}\text { Seth et. al (2016) } \\
-N_{u}\end{array}$} & \multicolumn{3}{c}{ Present results } \\
$N_{r} \downarrow Q_{H} \rightarrow N_{u}$ \\
\hline 2 & 1 & 3 & 5 & 1 & 3 & 5 \\
\hline 4 & 0.4586 & 0.6600 & 0.8159 & 0.4585 & 0.6601 & 0.8160 \\
\hline 6 & 0.3553 & 0.5112 & 0.6320 & 0.3553 & 0.5112 & 0.6320 \\
\hline & 0.3002 & 0.4321 & 0.5341 & 0.3001 & 0.4321 & 0.5341 \\
\hline
\end{tabular}

Table 1. Comparison of present results with those of Seth et. al (2016) when $P_{r}=0.71, R=0, t_{1}=2$ and $t=1.4$. 


\begin{tabular}{ccccccc}
\hline & \multicolumn{3}{c}{ Seth et. al (2016) } & \multicolumn{3}{c}{ Present results } \\
& \multicolumn{3}{c}{$-N_{u}$} & & & $-N_{u}$ \\
\cline { 2 - 7 }$t_{1} \downarrow N_{r} \rightarrow$ & 2 & 4 & 6 & 2 & 4 & 6 \\
\hline 1 & 0.8982 & 0.6957 & 0.5880 & 0.8980 & 0.6957 & 0.5880 \\
\hline 1.5 & 0.5988 & 0.4638 & 0.3920 & 0.5989 & 0.4638 & 0.3921 \\
\hline 2 & 0.4491 & 0.3479 & 0.2940 & 0.4491 & 0.3480 & 0.2940 \\
\hline
\end{tabular}

Table 2. Comparison of present results with those of Seth et. al (2016) when

$$
P_{r}=0.71, R=0, Q_{H}=3 \text { and } t=0.9 \text {. }
$$

\section{Graphical Results and discussion}

In order to determine the impact of various control parameters on the primary velocity $u(\eta, t)$, secondary velocity $w(\eta, t)$, temperature $\theta(\eta, t)$, concentration $\phi(\eta, t)$, primary shear stress $\tau_{x}$, secondary shear stress $\tau_{z}$, Nusselt number $N_{u}$ and Sherwood number $S_{h}$, numerical computations were done and depicted graphically in figures 2 to 12 and tables 3 to 6 . These results show the influence of the physical parameters on the flow fields.

Figures 2 to 7 were plotted to show the impact of $G_{r}, G_{m}, M, m, N_{r}, Q_{H}, R, K, S_{r}, \gamma$ and $S_{c}$ on the primary velocity $u(\eta, t)$ and secondary velocities $w(\eta, t)$. It is seen from Fig.2(a) that both primary and secondary velocities are getting accelerated with enhancement in $G_{r}$. Physically, increase in $G_{r}$ tends to enhance thermal buoyancy force, which cause to accelerates both $u$ and $w$ throughout the boundary layer. It is observed from Fig.2(b) that both $u$ and $w$ increase with increasing values of mass Grashof number $G_{m}$. Physically, increase in $G_{m}$ indicates smaller viscous effect than solutal buoyancy effect in the momentum equation, and consequently, causes to increase the fluid velocity in both primary and secondary flow directions throughout the boundary layer. It is perceived from Fig.3 (a) that fluid velocity decreased in the primary flow direction with an enhancement in $M$ whereas the reverse effect is observed on the secondary flow direction. Application of the transverse magnetic field always results in a resistive type force (Lorentz force) which is similar to the drag force and upon increasing the values of $M$, the drag force increases and tends to resist the fluid velocity significantly in the primary flow direction. Figure 3(b) reveals the impact of Hall parameter $m$ on the primary and secondary velocities. It is clear that Hall parameter tends to accelerate the fluid flow in both primary and secondary flow directions throughout the boundary layer. It can be seen from Fig.4 (a) that an increase in radiation parameter $N_{r}$ tends to enhance both primary and secondary velocities throughout the boundary layer. It is clear from Fig.4 (b) that increasing heat absorption parameter $Q_{H}$ tends to decelerate both velocity components. Physically, the presence of heat sink in the boundary layer absorbs energy which causes to decrease the fluid temperature as a result decreases the fluid velocity due to the buoyancy effect. The effects of radiation absorption parameter $R$ on both primary and secondary velocities are elucidated in Fig.5 (a). It is found that radiation absorption parameter tends to accelerate both velocity components throughout the boundary layer. It is clearly seen from Fig.5(b) that both primary and secondary velocities are increased due to increase in the permeability parameter $K$. Physically, increase in $K$ tends to decrease the resistance of the porous medium as a result increase the fluid velocity. 

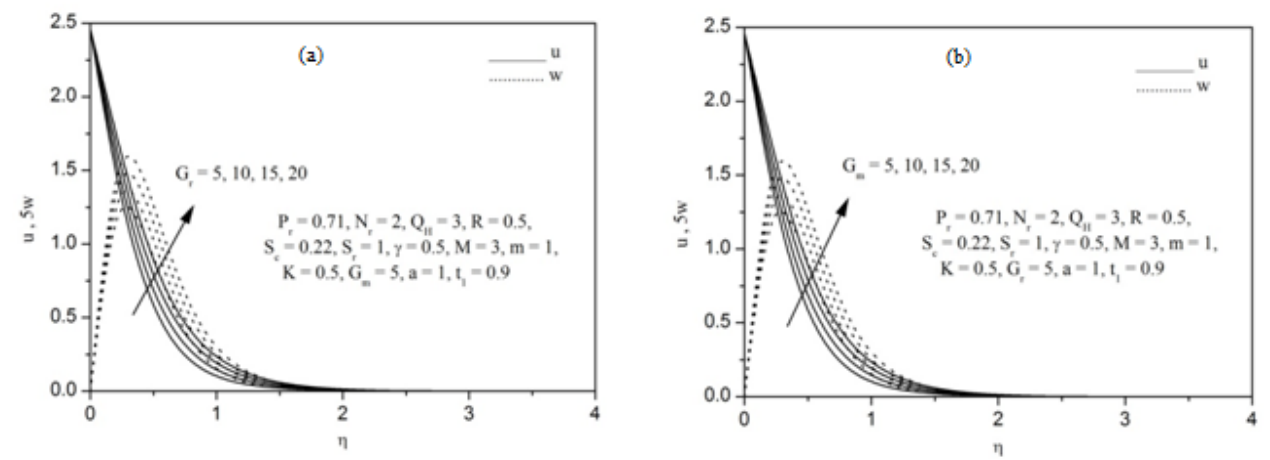

Fig. 2. Primary and secondary velocities $u$ and $w$ for different values of (a) $G_{r}$ (b) $G_{m}$.
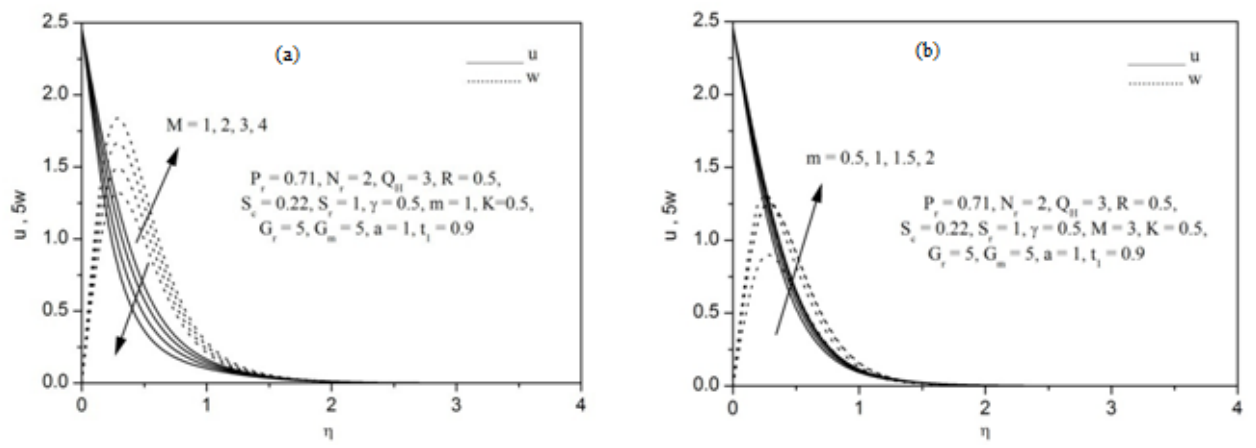

Fig. 3. Primary and secondary velocities $u$ and $w$ for different values of (a) $M$ (b) $m$.
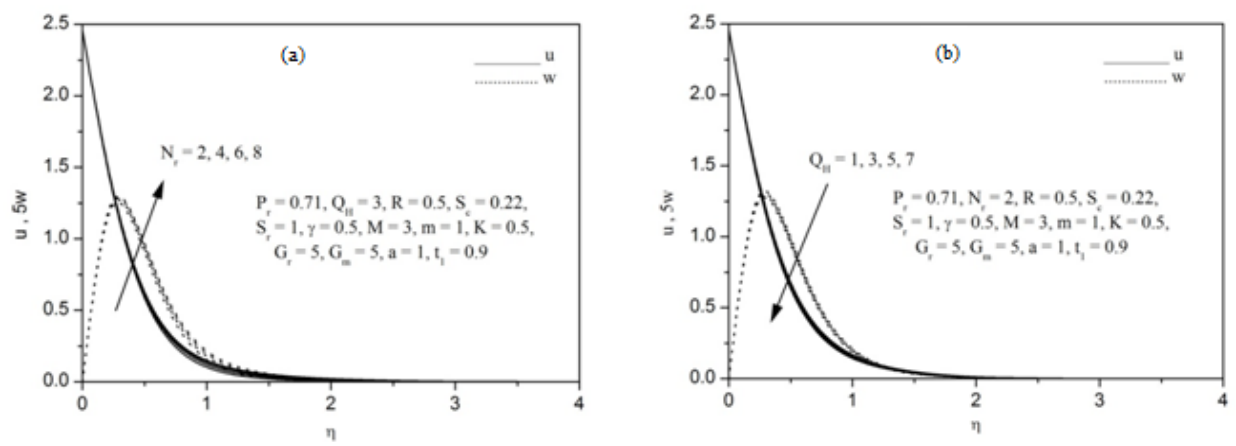

Fig. 4. Primary and secondary velocities $u$ and $w$ for different values of (a) $N_{r}$ (b) $Q_{H}$.

It is noticed from Fig. 6(a) that both primary and secondary velocities are increased throughout the boundary layer as the Soret number $S_{r}$ increased. It is seen from Fig.6 (b) that an increase in chemical reaction rate $\gamma$ tends to depreciate both $u$ and $w$ throughout the boundary 
layer region. It is found from Fig.7 that both $u$ and $w$ decreased throughout the boundary layer with $S_{c}$ increased due to the decrease in molecular diffusivity, which result in a decrease of the concentration and velocity boundary layer thickness.
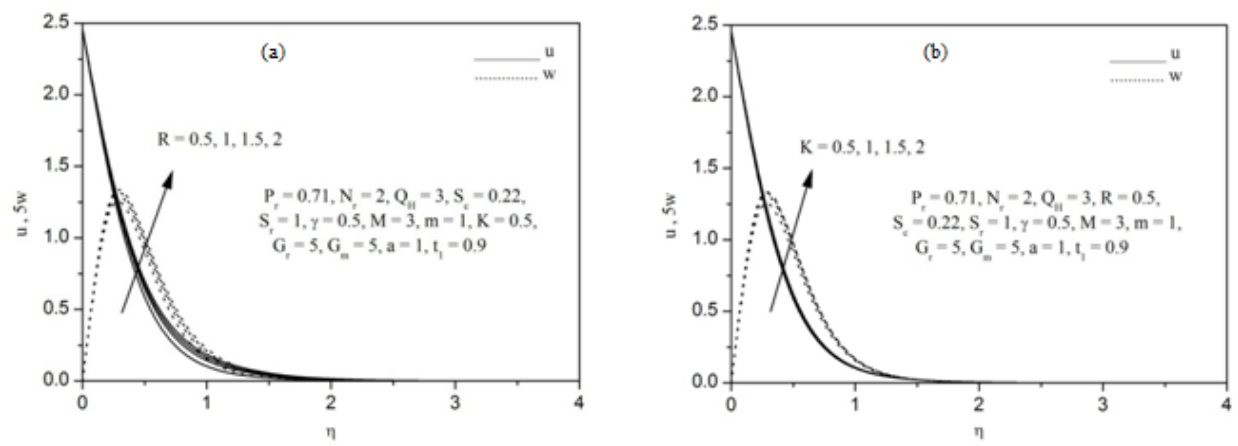

Fig. 5. Primary and secondary velocities $u$ and $w$ for different values of (a) $R$ (b) $K$.
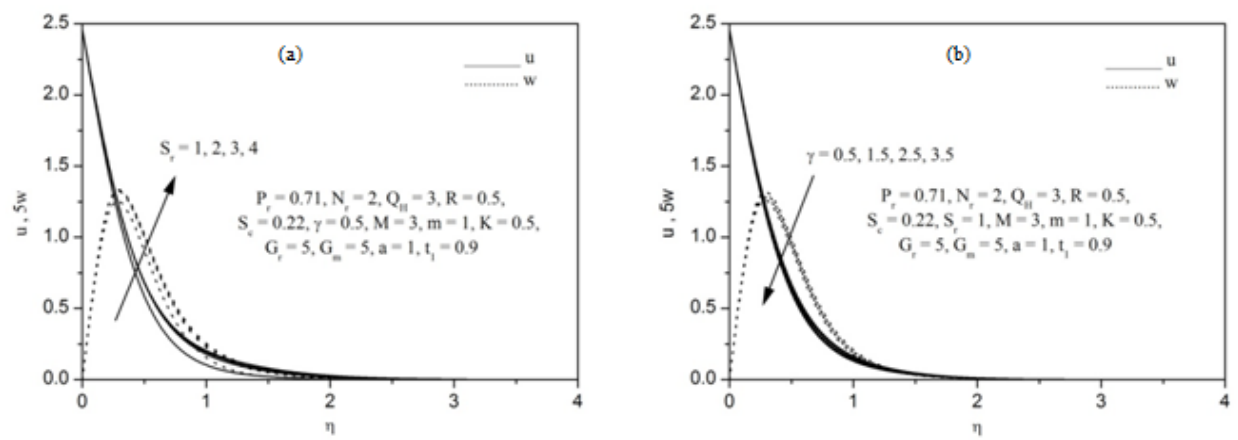

Fig. 6. Primary and secondary velocities $u$ and $w$ for different values of (a) $S_{c}$ (b) $\gamma$.

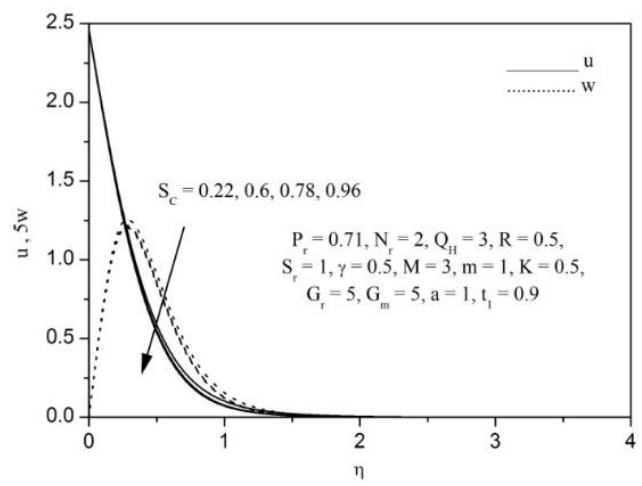

Fig. 7. Primary and secondary velocities $u$ and $w$ for different values of $S_{c}$. 
Figures 8 to 10 illustrates the temperature distribution $\theta(\eta, t)$ under the influence of $P_{r}$, $N_{r}, Q_{H}, R$ ant $t$ for both ramped and isothermal cases. It is observed from Fig.8(a) that the fluid temperature falls with an enhancement in Prandtl number $P_{r}$, because lower value of $P_{r}$ has more uniform temperature distribution across the thermal boundary layer as compared to higher $P_{r}$. It is clear from Fig.8(b) that the fluid temperature enhances in boundary layer as the radiation parameter $N_{r}$ increases. Figure 9(a) elucidates that the fluid temperature $\theta$ decreases with increasing values of heat absorption parameter $Q_{H}$. Physically, when heat absorption exist, thermal boundary layer is always starting to be thickened as result fluid temperature depreciate in the boundary layer. It can be noticed from Fig.9 (b) that an increase in the radiation absorption parameter $R$ causes improvement of the fluid temperature.
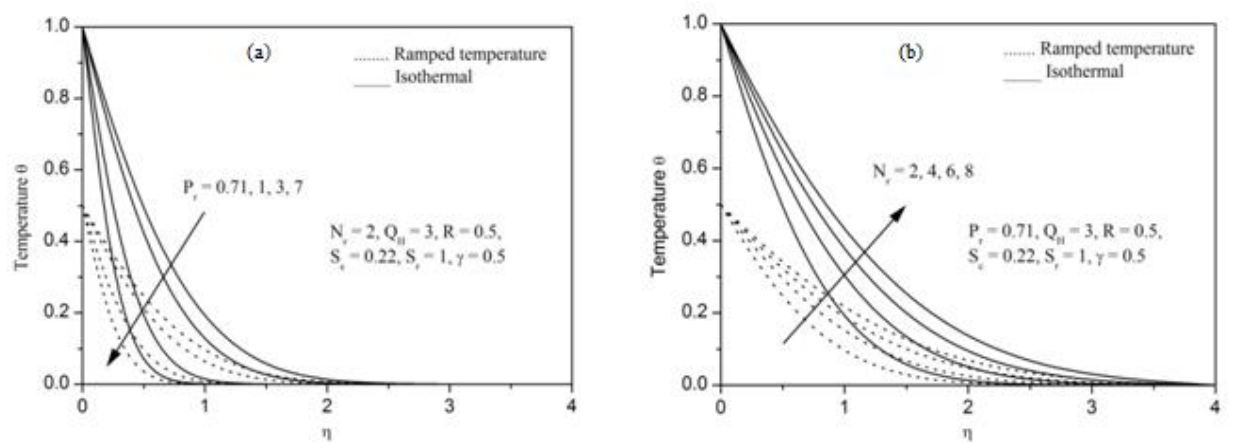

Fig. 8. Temperature profiles $\theta$ for different values of (a) $P_{r}$ (b) $N_{r}$.
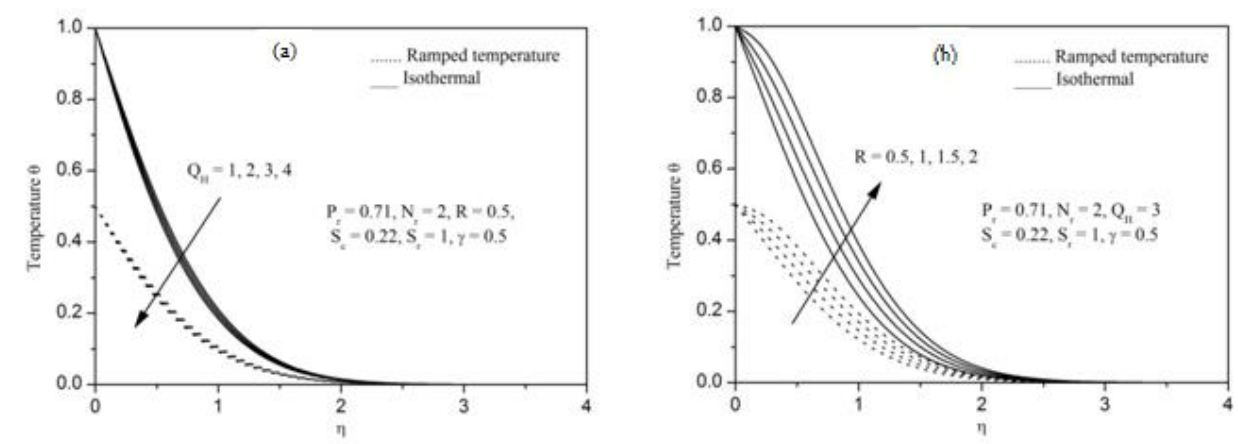

Fig. 9. Temperature profiles $\theta$ for different values of (a) $Q_{H}$ (b) $R$. 


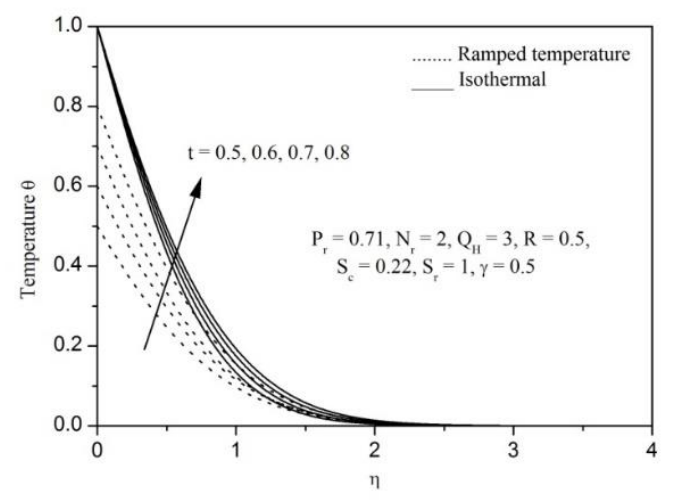

Fig. 10. Temperature profiles $\theta$ for different values of $t$.

The fluid temperature $\theta$ increase with the progression of time $t$ is seen in Fig.10. Further, when $t>t_{1}$, temperature becomes more uniform and it is equivalent to 1 , and when $0<t \leq t_{1}$, temperature takes the values of $t$ at the plate $(\eta=0)$ and tends to zero for large values of $\eta$, which is the clear verification of the boundary condition given in $(14 c)-(14 e)$. However, the nature of the fluid temperature is same when $0<t \leq t_{1}$ and $t>t_{1}$.

Figures 11 and 12 displays the concentration distribution $\phi(\eta, t)$ under the influence of $S_{c}, S_{r}, \gamma$ and $t$. It is perceived from Fig.11 (a) that the species concentration of the fluid decline when $S_{c}$ increased due to the decrease of concentration boundary layer thickness. The exactly reverse trend is noticed in the species concentration when the Soret number $S_{r}$ increased is seen in Fig.11 (b). It is found from Fig.12 (a) that the fluid concentration decreases with increasing values of chemical reaction parameter $\gamma$. Physically, large values of $\gamma$ tends to reduce the solutal boundary layer thickness and increase the mass transfer. As seen from Fig.12 (b), the fluid concentration increases with progression of time $t$. Further, it clearly verifies the boundary condition given in (14b) and (14e). Initially, species concentration $\phi$ takes the value 1 and afterward for large values of $\eta(\eta>0)$, it tends to zero with increase of $t$.
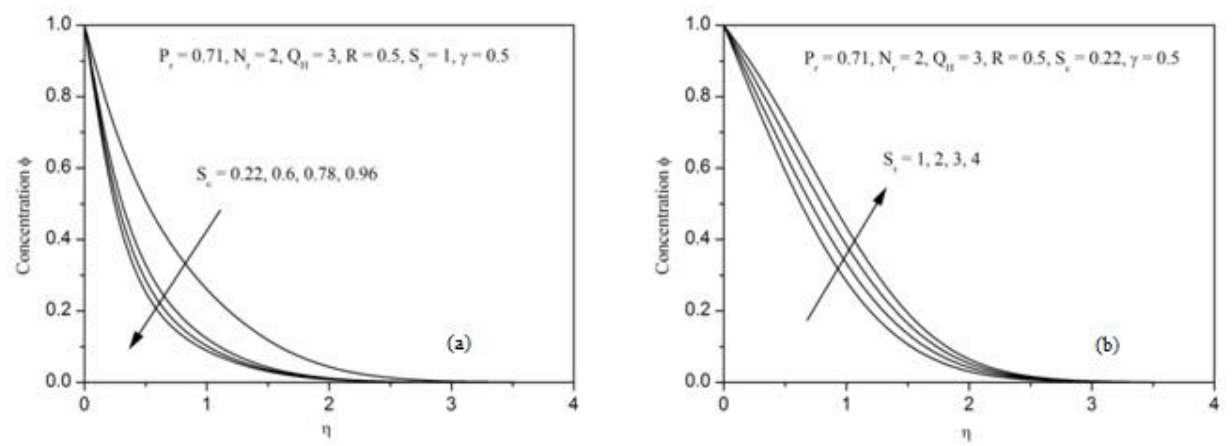

Fig. 11. Concentration profiles $\phi$ for different values of (a) $S_{c}$ (b) $S_{r}$. 

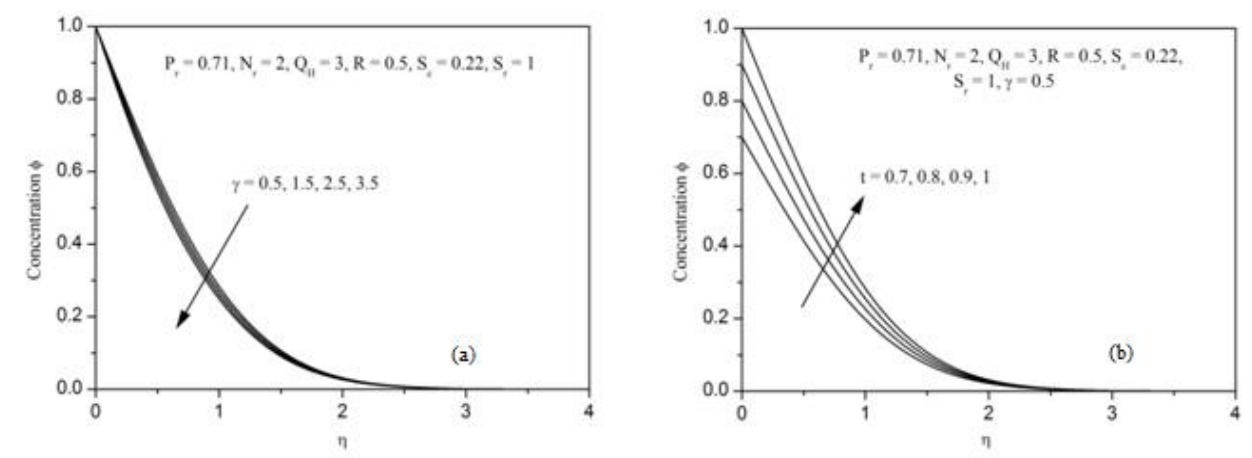

Fig. 12. Concentration profiles $\phi$ for different values of (a) $\gamma$ (b) $t$.

Tables 3 and 4 display the numerical values of primary and secondary shear stresses $\tau_{x}$ and $\tau_{z}$ for various values of $G_{r}, G_{m}, M, m, K, N_{r}, S_{c}, S_{r}, Q_{H}, R$ and $\gamma$. It is noticed that the primary shear stress $\tau_{x}$ is reducing when the values of $G_{r}, G_{m}, m, K, N_{r}, S_{c}$ and $R$ are increasing whereas it is enhanced when the values of $S_{c}, Q_{H}$ and $\gamma$ are increased and reverse effect is noticed on the secondary shear stress $\tau_{z}$. Both $\tau_{x}$ and $\tau_{z}$ increased when $M$ is increased.

\begin{tabular}{|c|c|c|c|c|c|c|c|}
\hline$G_{r}$ & $G_{m}$ & $M$ & $m$ & $K$ & $N_{r}$ & $-\tau_{x}$ & $-\tau_{z}$ \\
\hline 5 & & & & & & 4.047520 & 2.830288 \\
\hline 10 & 5 & 3 & 1 & 0.5 & 2 & 3.683592 & 3.013102 \\
\hline 15 & & & & & & 3.319664 & 3.195916 \\
\hline \multirow{3}{*}{5} & 5 & \multirow{3}{*}{3} & \multirow{3}{*}{1} & \multirow{3}{*}{0.5} & \multirow{3}{*}{2} & 4.047520 & 2.830288 \\
\hline & 10 & & & & & 3.642068 & 3.039034 \\
\hline & 15 & & & & & 3.236612 & 3.247778 \\
\hline \multirow{3}{*}{5} & \multirow{3}{*}{5} & 2 & \multirow{3}{*}{1} & \multirow{3}{*}{0.5} & \multirow{3}{*}{2} & 4.371152 & 3.339208 \\
\hline & & 3 & & & & 5.025116 & 3.744406 \\
\hline & & 4 & & & & 5.731348 & 4.145126 \\
\hline \multirow{3}{*}{5} & \multirow{3}{*}{5} & \multirow{3}{*}{3} & 0.5 & \multirow{3}{*}{0.5} & \multirow{3}{*}{2} & 4.379240 & 2.054190 \\
\hline & & & 1.5 & & & 3.800752 & 2.790364 \\
\hline & & & 2 & & & 3.648836 & 2.830288 \\
\hline \multirow{3}{*}{5} & \multirow{3}{*}{5} & \multirow{3}{*}{3} & \multirow{3}{*}{1} & 1 & \multirow{3}{*}{2} & 3.911052 & 2.938114 \\
\hline & & & & 1.5 & & 3.864532 & 2.975166 \\
\hline & & & & 2 & & 3.841080 & 2.993904 \\
\hline \multirow{3}{*}{5} & \multirow{3}{*}{5} & \multirow{3}{*}{3} & \multirow{3}{*}{1} & \multirow{3}{*}{0.5} & 4 & 4.006244 & 2.860790 \\
\hline & & & & & 6 & 3.981168 & 2.880428 \\
\hline & & & & & 8 & 3.963652 & 2.894632 \\
\hline
\end{tabular}

Table 3. Primary and secondary shear stresses $\tau_{x}$ and $\tau_{z}$ when $P_{r}=0.71, S_{c}=0.22$,

$$
S_{r}=1, Q_{H}=3, R=0.5, \gamma=0.5, a=1 \text { and } t=0.9 \text {. }
$$




\begin{tabular}{ccccccc}
\hline$S_{c}$ & $S_{r}$ & $Q_{H}$ & $R$ & $\gamma$ & $-\tau_{x}$ & $-\tau_{z}$ \\
\hline $\mathbf{0 . 6}$ & & & & & 4.133184 & 2.772414 \\
$\mathbf{0 . 7 8}$ & 1 & 3 & 0.5 & 0.5 & 4.156384 & 2.758568 \\
$\mathbf{0 . 9 6}$ & & & & & 4.174636 & 2.748216 \\
\hline & $\mathbf{2}$ & & & & 3.827424 & 2.942506 \\
0.22 & $\mathbf{3}$ & 3 & 0.5 & 0.5 & 3.799424 & 2.948858 \\
& $\mathbf{4}$ & & & & 3.784532 & 2.954238 \\
\hline & & $\mathbf{3}$ & & & 3.860816 & 2.915408 \\
0.22 & 1 & $\mathbf{5}$ & 0.5 & 0.5 & 3.911376 & 2.890058 \\
& & $\mathbf{7}$ & & & 3.961632 & 2.864812 \\
\hline & & & $\mathbf{1}$ & & 3.894828 & 2.901266 \\
0.22 & 1 & 3 & $\mathbf{1 . 5}$ & 0.5 & 3.823448 & 2.934930 \\
& & & $\mathbf{2}$ & & 3.755156 & 2.967602 \\
\hline \multirow{2}{*}{0.22} & & & & $\mathbf{1 . 5}$ & 3.893132 & 2.901538 \\
& & 3 & 0.5 & $\mathbf{2 . 5}$ & 3.961948 & 2.868422 \\
& & & & $\mathbf{3 . 5}$ & 4.029928 & 2.835632 \\
\hline
\end{tabular}

Table 4. Primary and secondary shear stresses $\tau_{x}$ and $\tau_{z}$ when $P_{r}=0.71, G_{r}=5$,

$$
G_{m}=5, M=3, m=1, K=0.5, N_{r}=2, a=1 \text { and } t=0.9 \text {. }
$$

Table 5 represents the numerical values of heat transfer rate at the plate $N_{u}$ for various values of $P_{r}, N_{r}, Q_{H}, R$ and $t$. It is found that for both kinds of thermal conditions, $N_{u}$ increases with increasing $P_{r}$ and $Q_{H}$ whereas it decreases with increasing $N_{r}$ and $R$. Further, in case of ramped temperature plate, $N_{u}$ gradually increases with progression of time whereas completely reverse trend takes place in case of isothermal plate. Table 6 exhibits the numerical values of mass transfer rate at the plate $S_{h}$ for various values of $S_{c}, S_{r}, \gamma$ and $t$. It is noticed that $S_{h}$ increases with an enhancement in $S_{c}, \gamma$ and $t$ whereas it decreases with increasing values of $S_{r}$.

\begin{tabular}{|c|c|c|c|c|c|c|}
\hline$P_{r}$ & $N_{r}$ & $Q_{H}$ & $R$ & $t$ & $\begin{array}{c}\text { ramped } \\
\text { temperature } \\
\text { plate } \\
-N_{u}\end{array}$ & $\begin{array}{l}\text { isotherma } \\
\text { plate } \\
-N_{u}\end{array}$ \\
\hline 1 & \multirow{3}{*}{2} & \multirow{3}{*}{3} & \multirow{3}{*}{0.5} & \multirow{3}{*}{0.5} & 0.537558 & 1.074776 \\
\hline 3 & & & & & 0.879124 & 1.758248 \\
\hline 7 & & & & & 1.230092 & 2.460180 \\
\hline \multirow{3}{*}{0.71} & 4 & \multirow{3}{*}{3} & \multirow{3}{*}{0.5} & \multirow{3}{*}{0.5} & 0.359716 & 0.719432 \\
\hline & 6 & & & & 0.306212 & 0.612428 \\
\hline & 8 & & & & 0.271300 & 0.542596 \\
\hline \multirow{3}{*}{0.71} & \multirow{3}{*}{2} & 2 & \multirow{3}{*}{0.5} & \multirow{3}{*}{0.5} & 0.437928 & 0.875856 \\
\hline & & 3 & & & 0.458072 & 0.916140 \\
\hline & & 4 & & & 0.477616 & 0.955228 \\
\hline \multirow{3}{*}{0.71} & \multirow{3}{*}{2} & \multirow{3}{*}{3} & 1 & \multirow{3}{*}{0.5} & 0.264024 & 0.528052 \\
\hline & & & 1.5 & & 0.169228 & 0.338456 \\
\hline & & & 2 & & 0.075880 & 0.151764 \\
\hline \multirow{3}{*}{0.71} & \multirow{3}{*}{2} & \multirow{3}{*}{3} & \multirow{3}{*}{0.5} & 0.6 & 0.549684 & 0.990184 \\
\hline & & & & 0.7 & 0.664824 & 0.949752 \\
\hline & & & & 0.8 & 0.732912 & 0.916140 \\
\hline
\end{tabular}

Table 5. Nusselt number $N_{u}$ when $S_{c}=0.22, S_{r}=1$ and $\gamma=0.5$. 


\begin{tabular}{ccccc}
\hline$S_{c}$ & $S_{r}$ & $\gamma$ & $t$ & $-S_{h}$ \\
\hline $\mathbf{0 . 6}$ & & & & 1.540896 \\
$\mathbf{0 . 7 8}$ & 1 & 0.5 & 1 & 1.706060 \\
$\mathbf{0 . 9 6}$ & & & & 1.855124 \\
\hline \multirow{2}{*}{0.22} & $\mathbf{2}$ & & & 0.577400 \\
& $\mathbf{3}$ & 0.5 & 1 & 0.473808 \\
& $\mathbf{4}$ & & & 0.370216 \\
\hline \multirow{2}{*}{022} & & $\mathbf{1 . 5}$ & & 0.725312 \\
& 1 & $\mathbf{2 . 5}$ & 1 & 0.768248 \\
& & $\mathbf{3 . 5}$ & & 0.809852 \\
\hline \multirow{2}{*}{0.22} & & & $\mathbf{0 . 7}$ & 0.476696 \\
& 1 & 0.5 & $\mathbf{0 . 8}$ & 0.544796 \\
& & & $\mathbf{0 . 9}$ & 0.612896 \\
\hline
\end{tabular}

Table 6. Sherwood number $S_{h}$ when $P_{r}=0.71, N_{r}=2, Q_{H}=3$ and $R=0.5$.

\section{Conclusions}

The present paper provides thermo-diffusion and Hall effects on unsteady MHD convective radiating, chemically reacting and heat absorbing fluid past an exponentially accelerated vertical porous plate with ramped temperature by finite element method. Results derived from this investigation can be summarized as:

1. Due to increase in $G_{r}, G_{m}, m, K, N_{r}, S_{r}$ and $R$ increases primary and secondary fluid velocities whereas expresses the reverse effect when $S_{c}, Q_{H}$ and $\gamma$ are increased.

2. Magnetic parameter $M$ tends to decelerates primary fluid velocity whereas reverse trend takes place on the secondary fluid velocity.

3. Temperature of the fluid enhances with rising values of $N_{r}, R$ and $t$ whereas opposite trend is noticed when $P_{r}$ and $Q_{H}$ are increased for both kind of thermal conditions.

4. Concentration of the fluid decline with enhancement in $S_{c}$ and $\gamma$ whereas reverse trend is observed when $S_{r}$ and $t$ are increased.

5. Primary shear stress $\tau_{x}$ getting reduced with increment in $G_{r}, G_{m}, m, K, N_{r}, S_{r}$ and $R$ whereas reverse phenomenon is noticed in case of secondary shear stress $\tau_{z}$.

6. Magnetic parameter $M$ causes to rise both $\tau_{x}$ and $\tau_{z}$.

7. Increase in $N_{r}$ and $R$ causes to depreciate in $N_{u}$ whereas reverse effect is noticed when $P_{r}$ and $Q_{H}$ are increased for both kind of thermal conditions.

8. In case of ramped temperature plate, $N_{u}$ gradually increases with increasing time whereas reverse trend takes place in case of isothermal plate.

9. Increase in $S_{c}, \gamma$ and $t$ tends to enhance in $S_{h}$ whereas reverse trend is observed when $S_{r}$ increased. 


\section{References}

Anand Rao J, Prabhakar Reddy B (2010). Heat and mass transfer of an unsteady MHD natural convection flow of a rotating fluid past a vertical porous plate in the presence of radiative heat transfer, J. Energy, Heat and Mass Transfer., 32, 223 - 241.

Bathe K J (1996). Finite Element Procedures, Prentice-Hall, New Jersey.

Das S, Sarkar B C, Jana R N (2013). Hall effect on MHD free convection boundary layer flow past a vertical flat plate, Meccanica, 48, 6, $1387-1398$.

Das S, Guchaitt S K, Jana R N, Makinde O D (2016). Hall effect on an unsteady magnetoconvection and radiative heat transfer past a porous plate, Alexandria Engineering Journal, $55,2,1321-1331$.

Das S, Jana R N, Ghosh S K (2016). Hall effect on unsteady MHD natural convective flow past an impulsively moving plate with ramped temperature and concentration, Ind. J. Pure and Appl. Phys., 54, $517-534$.

Makinde O D (2005). Free convection flow with thermal radiation and mass transfer past a moving vertical plate, Int. Comm. Heat and Mass Transfer., 21, 10, 1411-1419.

Mahmoud M A A (2009). Thermal radiation effects on unsteady MHD free convection flow past a vertical plate with temperature dependent viscosity, Canadian J. Chem. Engg., 87, 47-52.

Mahapatra N, Dash G C, Panda S, Aharya M (2010). Effects of chemical reaction on free convection flow through a porous medium bounded by a vertical surface, J. Engg. Phys. Thermo-Phys., 83, 1, $130-140$.

Makinde O D, Olanrewaju P O (2011). Unsteady mixed convection with Soret and Dufour effects past a porous plate moving through a binary mixture of chemically reacting fluid. Che. Engg. Comm., 198, 7, 920-938.

Mukhopadhyay S (2009). The effects of thermal radiation on unsteady mixed convection flow and heat transfer over a porous stretching surface in porous medium, Int. J. Heat and Mass Transfer., 52, 3261-3265.

Muralidhran M, Muthucumaraswamy R (2013). Radiation effects on linearly accelerated plate with variable mass diffusion in the presence of magnetic field, Appl. Math, Sci., 7 113, 5645 $-5656$.

Prabhakar Reddy B, Ananad Rao J (2011). Radiation and thermal diffusion effects on an unsteady MHD free convection mass transfer flow past an infinite vertical porous plate with Hall current and heat source, J. Engg. Phys. Thermo-Phys., 84, 6, 1369 - 1378.

Prakash J, Durga Prasad P, Kiran Kumar R V M S S, Varma S V K (2016). Diffusion thermo effects on MHD free convective radiative and chemically reactive boundary layer flow through a porous medium over a vertical plate, J. Comp. Appl. Res. Mech. Engg., 6, 2, 111 $-126$.

Prabhakar Reddy B, Sunzu J M (2018). Dufour and Soret effects on unsteady MHD free convection flow of viscous incompressible fluid past an infinite vertical porous plate in the presence of radiation, J. Serbian Soc. Com. Mech., 12, 1, 9-26.

Prabhakar Reddy B, Peter J (2019). Effects of chemical reaction on MHD flow past an impulsively started infinite vertical plate with variable temperature and diffusion in the presence of Hall current, J. Serbian Soc. Comp. Mech., 13, 1, 92 - 108.

Prabhakar Reddy B (2019). Radiation and chemical reaction effects on unsteady MHD free convection parabolic flow past an isothermal infinite vertical plate with viscous dissipation, Int. J Appl. Mech. Engg., 24, 2, 343 - 358.

Rajesh V, Varma S V K (2010). Radiation effects on MHD flow through a porous medium with variable temperature and mass diffusion, Int. J. Appl. Math and Mech., 6, 1, 39-57.

Raju M C, Varma S V K, Ananad Reddy N (2011). Hall current effects on unsteady MHD flow between stretching sheet and an oscillatory porous upper parallel plate with constant suction, Thermal Science., 15, 2, $45-48$. 
Rajesh V (2012). Effects of mass transfer on flow past an impulsively started infinite vertical plate with Newtonian heating and chemical reaction, J. Engg. Phys. Thermo-Phys., 85, 1, 221-228.

Rajput U S and Kanaujia N (2016). MHD flow past a vertical plate with variable temperature and mass diffusion in the presence of Hall current, Int. J. Appl. Sci. Engg., 14, 2, 115 - 123.

Reddy J N (1985). An Introduction to the Finite Element Method, McGraw-Hill, New York

Saha I K, Siddiqa S, Hossain M A (2011). Effect of Hall current on MHD natural convection flow from vertical permeable flat plate with uniform surface heat flux, Appl. Math. Mech., Engl. $32,1127-1146$.

Seth G S, Kumbhakar B, Sharma R (2016). Unsteady MHD free convection flow with Hall effect of a radiating and heat absorbing fluid past a moving vertical plate with variable ramped temperature, J. Egyptian Math. Soc, 24, 471-478.

Seth G S, Bhattacharyya A, Tripathi R (2017). Effect of Hall current on MHD natural convection heat and mass transfer flow of rotating fluid past a vertical plate with ramped wall temperature, Frontiers in Heat and Mass Transfer., 9, 21, 1- 12.

Shanker R T, Gnaneshwar R M (2007). Radiation effects on MHD flow past an impulsively started infinite vertical plate through a porous medium with variable temperature and mass diffusion, J. Pure and Appl. Phys., 19, 3, 191-200.

Shanker B, Prabhakar Reddy B, Anand Rao J (2010). Radiation and mass transfer effects on an unsteady MHD free convective fluid flow embedded in a porous medium with heat generation/absorption, Ind. J. Pure and Appl. Phys., 48, 157 - 167.

Shateyi S, Motsa S (2011). Unsteady magneto-hydrodynamic convective heat and mass transfer past an infinite vertical plate in a porous medium with radiation, heat generation/absorption and chemical reaction, Advanced Topics in Mass Transfer., $145-162$.

Sharma B H, Jha A K, Chaudary R C (2007). Hall effects on MHD mixed convective flow of a viscous incompressible fluid past a vertical porous plate immersed in a porous medium with heat source/sink, Rom. J. Phys., 52, 5-7, $487-503$.

Siva Kumar N, Rushi Kumar B, Vijaya Kumar A G (2016). Thermal diffusion and chemical reaction effects on unsteady flow past a vertical porous plate with heat source in slip flow regime, J. Naval Arch. Marine Engg., 13, 1, 51-62.

Soundalgekhar V M, Takhar H S (1993). Radiation effects on free convection flow past a semiinfinite vertical plate, Modeling Measurement and Control, B, 51, 31-40.

Takhar H S, Gorla R S, Soundalgekhar V M (1996). Radiation effects on MHD free convection flow of a radiating gas past a semi-infinite vertical plate, Int. J. Num. Heat and Fluid Flow, $6,2,77-83$.

Venkateswarlu M, Ramana Reddy G V, Venkata Lakshmi D (2013). Effects of chemical reaction and heat generation on MHD boundary layer flow of a moving vertical plate with suction and viscous dissipation, Engineering International., 1, 1, 27 - 38.

Venkateswarlu M, Padma P (2015). Unsteady MHD free convective heat and mass transfer in a boundary layer flow past a vertical permeable channel with thermal radiation and chemical reaction, Procedia Engineering., 127, $791-799$.

Venkateswarlu M, Venkata Lakshmi D (2017). Thermal diffusion, Hall current and chemical reaction effects on unsteady MHD natural convection flow past a vertical plate, U. P. B. Sci. Bull, Series D, Mechanical Engineering journal., 6, 363 - 371. 